\title{
State Constraints in the Linear Regulator Problem: Case Study ${ }^{1}$
}

\author{
A. L. Dontchev ${ }^{2}$ AND I. V. Kolmanovsky ${ }^{3}$
}

Communicated by T. L. Vincent

\begin{abstract}
In this paper, we consider the problem of minimum-norm control of the double integrator with bilateral inequality constraints for the output. We approximate the constraints by piecewise linear functions and prove that the Lagrange multipliers associated with the state constraints of the approximating problem are discrete measures, concentrated in at most two points in every interval of discretization. This allows us to reduce the problem to a convex finite-dimensional optimization problem. An algorithm based on this reduction is proposed and its convergence is examined. Numerical examples illustrate our approach. We also discuss regularity properties of the optimal control for a higherdimensional state-constrained linear regulator problem.
\end{abstract}

Key Words. Linear-quadratic problems, double integrators, state constraints, obstacle avoidance, finite-dimensional approximations.

\section{Introduction}

We consider the minimum-norm problem for the double integrator with bilateral state constraints as follows:

$$
\begin{array}{ll}
\min & \|u\|, \\
\text { s.t. } & \ddot{x}(t)=u(t), \\
& x(a)=y_{a}, \quad \dot{x}(a)=s_{a}, \quad x(b)=y_{b}, \quad \dot{x}(b)=s_{b}, \\
& g(t) \leq x(t) \leq f(t), \quad \text { for all } t \in[a, b], u \in L^{2}[a, b],
\end{array}
$$

\footnotetext{
'The first author was supported by the National Science Foundation, Grant No. DMS-9404431. The second author was supported by a François-Xavier Bagnoud Doctoral Fellowship and by NSF Grants DMS-9404431 and MSS-9114630.

${ }^{2}$ Associate Editor, Mathematical Reviews, Ann Arbor, Michigan.

${ }^{3} \mathrm{PhD}$ Student, Department of Aerospace Engineering, University of Michigan, Ann Arbor, Michigan.
} 
where $a, b, y_{a}, y_{b}, s_{a}, s_{b}$ are given numbers and $\|\cdot\|$ denotes the $L^{2}$ norm in the interval $[a, b]$. We assume that $f$ and $g$ are given functions that are continuous in the interval $[a, b]$ and such that $g(t)<f(t)$ for all $t \in[a, b]$ and $g(a)<y_{a}<f(a), g(b)<y_{b}<f(b)$; then, there exists a $C^{2}$ function $p$ satisfying the boundary conditions (1c) and such that $g(t)<p(t)<f(t)$. Hence, the set of feasible controls is not empty; since it is a closed and convex subset of $L^{2}[a, b]$, there exists a unique solution of problem (1). Moreover, by the existence of an interior trajectory, it follows that the first-order Lagrange optimality conditions are in the normal form; see, e.g., Refs. 1-2.

The double integrator is a classical example in optimal control which is often used to describe the motion of a car or trolley along a horizontal track without friction. The bilateral state constraints in problem (1) may be interpreted as arising from the presence of two other cars, on both sides of the controlled car, whose positions in time are given by the functions $g$ and $f$.

In this paper, we provide a nonstandard reduction of problem (1) to finite dimensions based on approximation of the constraints by functions that are piecewise extremal arcs. We approximate problem (1) in the following way. Let $a=t_{0}<t_{1}<\cdots<t_{n}=b$ be a partition of the interval $[a, b]$, and let $e$ and $d$ be two piecewise linear and continuous approximations of $g$ and $f$ respectively across the grid $\left\{t_{i}\right\}$ such that $e(t)<d(t)$ for all $t \in[a, b]$. Let $\epsilon$ be a fixed sufficiently small and positive number, e.g.,

$$
\epsilon<(1 / 4) \min _{t \in[a, b]}(d(t)-e(t)) .
$$

Consider the following problem:

$$
\begin{array}{ll}
\min & J(u, s, y)=\|u\|, \\
\text { s.t. } & \ddot{x}(t)=u(t), \\
& x(a)=y_{a}, \quad \dot{x}(a)=s_{a}, \quad x(b)=y_{b}, \quad \dot{x}(b)=s_{b}, \\
& e(t) \leq x(t) \leq d(t), \quad \text { for all } t \in[a, b], \\
& x\left(t_{i}\right)=y_{i}, \quad \dot{x}\left(t_{i}\right)=s_{i}, \quad i=1,2, \ldots, n-1, \\
& e\left(t_{i}\right)+\epsilon \leq y_{i} \leq d\left(t_{i}\right)-\epsilon, \quad i=1,2, \ldots, n-1, \\
& s=\left(s_{1}, s_{2}, \ldots, s_{n-1}\right) \in \mathbb{R}^{(n-1)}, \\
& y=\left(y_{1}, y_{2}, \ldots, y_{n-1}\right) \in \mathbb{R}^{(n-1)}, \quad u \in L^{2}[a, b] .
\end{array}
$$

Problem (2) can be rewritten as a two-stage minimum problem. Let

$$
y_{0}=y_{a}, \quad s_{0}=s_{a}, \quad y_{n}=y_{b}, \quad s_{n}=s_{b} .
$$


For fixed $s_{i}$ and $y_{i}, i=0,1, \ldots, n$, we first solve the problem

$$
\begin{array}{ll}
\min & I(u)=\|u\|, \\
\text { s.t. } & \ddot{x}(t)=u(t), \quad x\left(t_{i}\right)=y_{i}, \quad \dot{x}\left(t_{i}\right)=s_{i}, \quad i=0,1, \ldots, n, \\
& e(t) \leq x(t) \leq d(t), \quad \text { for all } t \in[a, b], u \in L^{2}[a, b] .
\end{array}
$$

This problem can be viewed as a constrained best approximation problem with mixed interpolation conditions. In a previous paper (Ref. 3), a Lagrange duality result for a related problem is established. Based on an extension of this result, in Section 2 we show that the unique optimal control of this problem is a piecewise linear function that can be computed in a finite number of steps. Let

$$
\Psi(s, y)=\min I(u)
$$

be the value (marginal) function of problem (3). In Section 4, we prove that this function is convex, coercive in $s$ uniformly in $y,(1 / 2)$-Lipschitz continuous, and has a unique minimum. The solution of (2) can be then obtained by solving the finite-dimensional convex programming problem

$\min \Psi(s, y)$,

s.t. $\quad s \in \mathbb{R}^{n-1}, \quad e\left(t_{i}\right)+\epsilon \leq y_{i} \leq d\left(t_{i}\right)-\epsilon, \quad i=1,2, \ldots, n-1$.

In Section 3, we study the convergence of the optimal solution of problem (2) for $\epsilon \rightarrow 0$ and when the piecewise linear approximations $e$ and $d$ of the constraints converge to $g$ and $f$ respectively. In Section 5, we discuss some generalizations, while in Section 6 we present numerical examples.

The approach presented in this paper may be useful for optimal control of mechanical systems in the presence of obstacles. As an example, consider a planar Cartesian manipulator (a factory crane) which moves in the plane, with constant velocity along the $x$-axis while the motion of the manipulator along the $y$-axis is subject to control. The problem of transferring the manipulator from one point to another avoiding given obstacles and spending minimum energy may be described with the help of the state-constrained double integrator considered in this paper. Another possible application of the results obtained in this paper is planning a highway in a specified region (with lakes, mountains, etc.), where the minimization of the second norm corresponds to minimizing the curvature of the highway.

Standard computational methods for optimal control problems are based on discretization in time usually combined with penalty function techniques; see, e.g., Refs. 4-8. Apparently, such an approach is justified when nonlinear higher-dimensional problems are to be solved. In the case of state 
constraints, the error analysis of discrete approximations may be considerably involved because of the implicit character of the constraints; see Ref. 9. In this paper, we follow a different path; considering a relatively simple problem, we choose an approximation on the basis of the properties of the solution. The key observation in our analysis is given in Theorem 2.1, where we show that the optimal control of problem (2) is a piecewise-linear function in time. For more general problems, there are only partial results on regularity of optimal controls. Hager (Ref. 10) considered a linear-convex optimal control problem with state and control inequality constraints proving that, under a certain linear independence condition for the active constraints, the optimal control is Lipschitz continuous in time. A more recent study of regularity properties of the measures representing the Lagrange multipliers for various state-constrained problems is given in a book by Dikusar and Milyutin (Ref. 11). In Section 7 (Appendix), we discuss the regularity properties of the optimal control for a higher-dimensional stateconstrained linear regulator problem, related to possible extensions of the approach of the present paper.

\section{Regularity of the Solution}

Consider problem (3) with fixed $s$ and $y$ such that $e\left(t_{i}\right)<y_{i}<d\left(t_{i}\right)$, and let $u^{*}$ be the optimal control, which exists and is unique. In this section, we show that the corresponding optimal output $x^{*}$ is a $C^{1}$ piecewise-cubic polynomial with a special structure. First, we need some terminology.

The points $t_{i}, i=0,1, \ldots, n$, are called fixed knots. Given an interval $\left[t_{i}, t_{i+1}\right]$, we say that the point $\tau \in\left(t_{i}, t_{i+1}\right)$ is a single touching point on the constraint $e$ if $x^{*}(\tau)=e(\tau)$ and $e(t)<x^{*}(t)<d(t)$ for all $t \neq \tau, t \in\left[t_{i}, t_{i+1}\right]$. An interval $\left[\tau_{1}, \tau_{2}\right] \subset\left(t_{i}, t_{i+1}\right), \tau_{1}<\tau_{2}$, is a subarc on the constraint $e$ if $x^{*}(t)=e(t)$ for all $t \in\left[\tau_{1}, \tau_{2}\right]$ and $e(t)<x^{*}(t)<d(t)$ for all $t \in\left[t_{i}, \tau_{1}\right) \cup\left(\tau_{2}, t_{i+1}\right]$. A touching point and a subarc for the upper constraint $d$ are defined in the same way. A pair $\left(\tau_{1}, \tau_{2}\right)$, with $\tau_{1}, \tau_{2} \in\left(t_{i}, t_{i+1}\right)$, is a touching pair if $x^{*}\left(\tau_{1}\right)=e\left(\tau_{1}\right)$ and $f\left(\tau_{2}\right)=d\left(\tau_{2}\right)$, and $e(t)<x^{*}(t)<d(t)$ for all $t \in\left[t_{i}, t_{i+1}\right], t \neq \tau_{1}, t \neq \tau_{2}$. The touching points, single or in a touching pair, and the ends of a subarc are called contact points.

Theorem 2.1. The unique optimal control $u^{*}$ of problem (3) is a piecewise-linear function which may be discontinuous only at the fixed knots $t_{i}$, $i=1, \ldots, n-1$. In every interval $\left(t_{i}, t_{i+1}\right)$, there are at most two contact points where the optimal output $x^{*}$ reaches or leaves the constraints. The optimal control $u^{*}$ is linear and $x^{*}$ is a cubic polynomial in every interval 


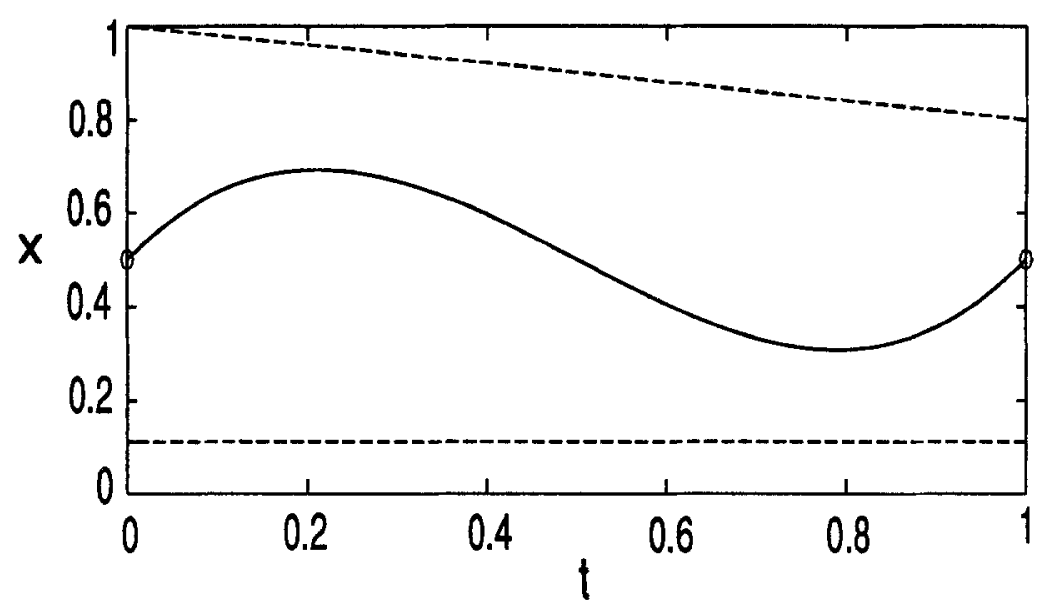

Fig. 1a. Possible location of contact points in $\left[t_{i}, t_{i+1}\right]$, according to Theorem 2.1. Case of no contact points.

which does not contain a fixed knot $t_{i}$ or a contact point. Moreover, in every interval $\left[t_{i}, t_{i+1}\right]$, the following cases are possible:

Case 1. The constraints are not active (no contact points).

Case 2. A single touching point occurs on one of the constraints (one contact point).

Case 3. A subarc occurs on one of the constraints (two contact points).

Case 4. A touching pair (two contact points) takes place.

These cases are illustrated in Figs. 1a-1d.

Lemma 2.1. There exist real numbers $\lambda_{i}$ and $k_{i}, i=0, \ldots, n$, and nonnegative regular measures $\mu_{1}$ and $\mu_{2}$, supported on the sets $T_{1}=\{t \in[a, b]$ : $\left.x^{*}(t)=e(t)\right\}$ and $T_{2}=\left\{t \in[a, b]: x^{*}(t)=d(t)\right\}$, respectively, such that

$$
u^{*}(t)=\int_{t}^{b} p_{1}(s) d s+\int_{t}^{b} d \sum_{i=0}^{n} \kappa_{i}\left(t-t_{i}\right)_{+}^{\prime},
$$

where

$$
p_{1}(t)=-\int_{1}^{b} d\left[\sum_{i=0}^{n} \lambda_{i}\left(t-t_{i}\right)_{+}^{\prime}+\mu_{2}-\mu_{1}\right] .
$$




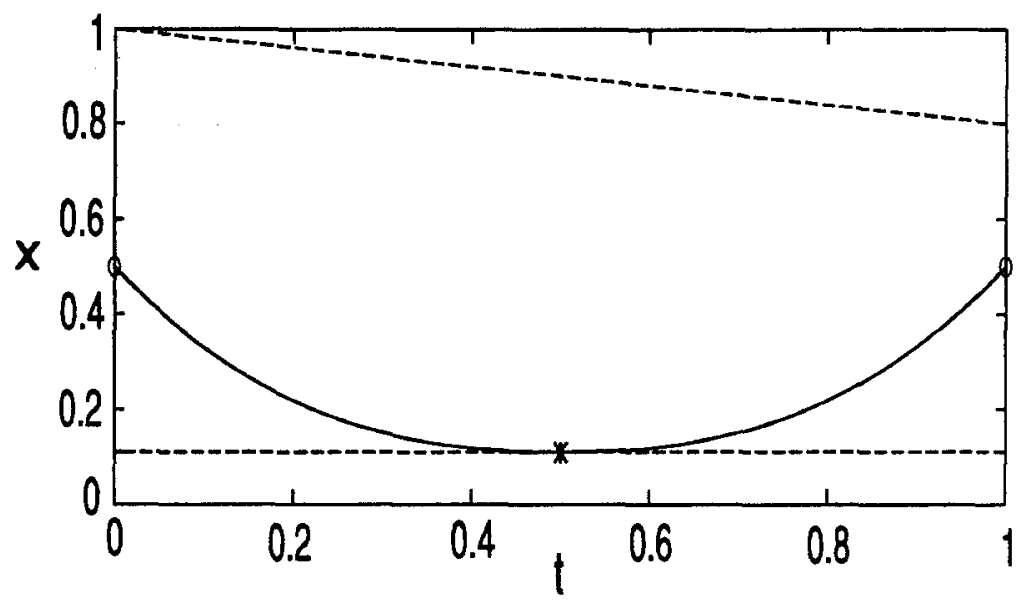

Fig. 1b. Possible location of contact points in $\left[t_{i}, t_{i+1}\right]$, according to Theorem 2.1. Case of a single touching point.

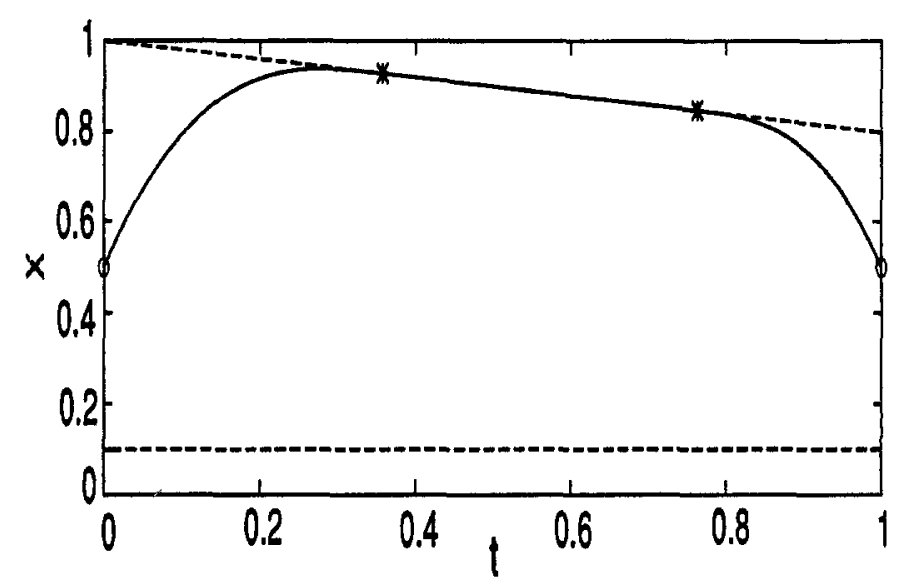

Fig. Ic. Possible location of contact points in $\left[t_{i}, t_{i+1}\right]$, according to Theorem 2.1. Case of a subarc.

Proof. The proof of the lemma is completely analogous to the proof of Lemma 1 in Ref. 3, where a constrained interpolation problem is studied. It can be also derived by the proof of (but does not follow directly from) the general first-order conditions for state-constrained problems given in Ref. 2. We note that the constants $\lambda_{i}, i=1,2, \ldots, n$, are the Lagrange multipliers corresponding to the conditions $x\left(t_{i}\right)=y_{i}, i=0, \ldots, n$; the constants $\kappa_{i}, i=0, \ldots, n$, are the Lagrange multipliers corresponding to the conditions $\dot{x}\left(t_{i}\right)=s_{i}, i=0, \ldots, n$; and the nonnegative measures $\mu_{j}$, 


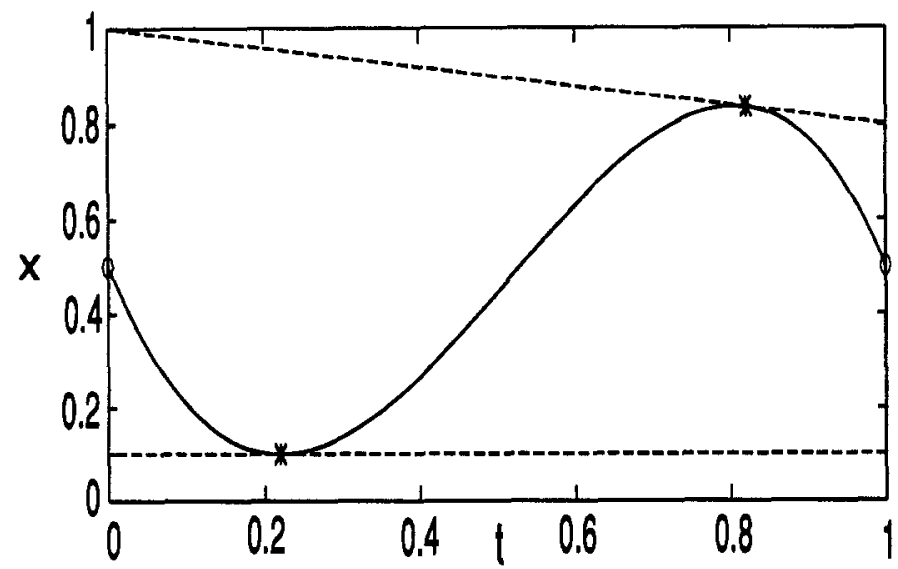

Fig. 1d. Possible location of contact points in $\left[t_{i}, t_{i+1}\right]$, according to Theorem 2.1. Case of a touching pair.

$j=1,2$, are the Lagrange multipliers corresponding to the inequality constraints.

Proof of Theorem 2.1. Lemma 2.1 implies that the optimal output $x^{*}$ is $C^{2}$ in every interval $\left(t_{i}, t_{i+1}\right)$ and is a cubic polynomial in every subinterval of $\left[t_{i}, t_{i+1}\right]$ where $e(t)<x^{*}(t)<d(t)$. Moreover, if $\tau$ is a contact point in $\left(t_{i}, t_{i+1}\right)$ where $x^{*}(\tau)=e(\tau)$, then $\dot{u}^{*}(\tau-0) \leq \dot{u}^{*}(\tau+0)$; if $x^{*}(\tau)=d(\tau)$, then $\dot{u}^{*}(\tau-0) \geq \dot{u}^{*}(\tau+0)$. Let $\tau_{1}, \tau_{2} \in\left(t_{i}, t_{i+1}\right)$, for some $i$, be such that $x^{*}\left(\tau_{1}\right)=$ $d\left(\tau_{1}\right)$ and $x^{*}\left(\tau_{2}\right)=d\left(\tau_{2}\right)$. Since $x^{*}(t) \leq d(t)$ and $x^{*} \in C^{1}$, then $\dot{x}^{*}\left(\tau_{1}\right)=\dot{d}\left(\tau_{1}\right)$ and $\dot{x}\left(\tau_{2}\right)=\dot{d}\left(\tau_{2}\right)$. The control

$$
\tilde{u}(t)= \begin{cases}0, & \text { if } t \in\left[\tau_{1}, \tau_{2}\right] \\ u^{*}(t), & \text { otherwise }\end{cases}
$$

is feasible and satisfies $\|\tilde{u}\| \leq\left\|u^{*}\right\|$, since $d$ is linear. Thus, $u^{*}(t)=\tilde{u}(t)$ and $x^{*}(t)=d(t)$ for $t \in\left[\tau_{1}, \tau_{2}\right]$. The function $x^{*}$ is continuous; hence, the set $\left\{t \in\left[t_{i}, t_{i+1}\right]: x^{*}(t)=d(t)\right\}$ is closed; thus, it has a minimal and a maximal element. The same conclusion holds for the other constraint. Summarizing, in every interval $\left[t_{i}, t_{i+1}\right]$, each of the constraints can be active no more than once, either in an interval whose endpoints are contact points, or in a single point which is a contact point.

We will prove that, if for some $\left[t_{i}, t_{i+1}\right]$ one of the constraints is active in a proper interval (with length $>0$ ), then the other constraint is nonactive in $\left[t_{i}, t_{i+1}\right]$. Let $\tau_{1}, \tau_{2}$ be two contact points, with $\tau_{1}, \tau_{2} \in\left[t_{i}, t_{i+1}\right], \tau_{1}<\tau_{2}$; let $\tau_{1}$ be the right end of a proper interval where the lower constraint $e$ is active; and let $\tau_{2}$ be the left end of an interval or a single point where the 
upper constraint $d$ is active; that is, $e(t)<x^{*}(t)<d(t)$, for $t \in\left(\tau_{1}, \tau_{2}\right)$. From Lemma 2.1, $u^{*}$ is continuous in $\left(t_{i}, t_{i+1}\right)$. The Taylor expansion in $t \in\left[\tau_{1}, \tau_{2}\right]$ gives us

$$
\begin{aligned}
x^{*}(t) & =x^{*}\left(\tau_{2}\right)+\dot{x}^{*}\left(\tau_{2}\right)\left(t-\tau_{2}\right)+u^{*}(\bar{\tau})\left(t-\tau_{2}\right)^{2} / 2 \\
& =d(t)+u^{*}(\bar{\tau})\left(t-\tau_{2}\right)^{2} / 2,
\end{aligned}
$$

for some $\bar{\tau} \in\left[t, \tau_{2}\right]$. Taking into account that $x^{*}(t)<d(t)$ for $t \in\left(\tau_{1}, \tau_{2}\right)$, we conclude that $u^{*}(\bar{\tau})<0$ for any $\bar{\tau}$ arbitrarily close to and less than $\tau_{2}$. Hence, $u^{*}\left(\tau_{2}\right) \leq 0$. On the other hand, $u^{*}\left(\tau_{1}\right)=0$, since $\tau_{1}$ is the right end of an interval where $u^{*}=0$. From Lemma 2.1, $\dot{u}^{*}$ has a jump upward at $\tau_{1}$. Hence, the linear function $u^{*}(t)$ is strictly increasing for $\tau_{1}<t<\tau_{2}$ and $u^{*}\left(\tau_{1}\right)=0$; thus, $u^{*}\left(\tau_{2}\right)>0$. The contradiction obtained implies that such a location of contact points is impossible. The proofs for the remaining cases are completely analogous. Hence, in any $\left[t_{i}, t_{i+1}\right]$, both constraints may be active only in a touching pair. This proves the theorem.

It turns out that, when we minimize with respect to $y$ and $s$ in problem (2), the optimal control becomes more regular.

Corollary 2.1. The optimal control $u_{*}$ of problem (2) satisfies all the regularity conditions in Theorem 2.1 ; moreover, $u_{*}$ is a continuous function in the whole interval $[a, b]$, and the optimal output $x_{*}$ is a $C^{2}$ piecewisecubic polynomial. If, in some interval $\left[t_{i}, t_{i+1}\right], x_{*}$ has a subarc on one of the constraints, then $x_{*}$ has no subarcs on the other constraint in $\left[t_{i-1}, t_{i+2}\right]$.

Proof. The solution $\left(u_{*}, x_{*}\right)$ of problem (2) is also a solution of the following problem:

$$
\begin{array}{ll}
\min & \|u\|, \\
\text { s.t. } & \ddot{x}(t)=u(t), \quad x\left(t_{i}\right)=x_{*}\left(t_{i}\right), \quad i=0,1, \ldots, n, \\
& e(t) \leq x(t) \leq d(t), \quad \text { for all } t \in[a, b], u \in L^{2}[a, b] .
\end{array}
$$

In Ref. 3, it is shown in the context of approximation theory that problem (5) has a unique continuous and piecewise linear optimal control $u_{*}$, and (compare with Lemma 2.1) there exist real numbers $l_{i}, i=0, \ldots, n$, and nonnegative regular measures $\mu_{1}$ and $\mu_{2}$ supported on the sets

$$
\begin{aligned}
& T_{1}=\left\{t \in[a, b]: x_{*}(t)=e(t)\right\}, \\
& T_{2}=\left\{t \in[a, b]: x_{*}(t)=d(t)\right\},
\end{aligned}
$$


respectively, such that

$$
\dot{u}_{*}(t)=l_{i}+\int_{t}^{b} d\left(\mu_{1}-\mu_{2}\right), \quad \text { for a.e. } t \in\left[t_{i}, b\right], \quad i=0, \ldots, n .
$$

By repeating the arguments used in the proof of Theorem 2.1, one can show that the solution to problem (5) satisfies all the regularity conditions of Theorem 2.1.

We now prove that, if $x_{*}$ has a subarc in $\left[t_{i}, t_{i+1}\right]$ on one of the constraint, say $e$, then $x_{*}$ has no subarcs in $\left[t_{i-1}, t_{i+2}\right]$ on the other constraint. Let $\tau_{1} \in\left[t_{i}, t_{i+1}\right]$ be the right end of a subarc on the constraint $e$, and let $\tau_{2} \in\left[t_{i+1}, t_{i+2}\right]$ be the right end of a subarc on the constraint $d$. Then, $u_{*}\left(\tau_{1}\right)=0=u_{*}\left(\tau_{2}\right)$ and, since $\tau_{1}$ and $\tau_{2}$ are contact points, $u_{*}(t) \geq 0$ for $t>\tau_{1}$ and $t$ near $\tau_{1}, u_{*}(t) \leq 0$ for $t<\tau_{2}$ and $t$ near $\tau_{2}$. But $u_{*}$ is piecewise linear and continuous with one knot $t_{i+1}$ between $\tau_{1}$ and $\tau_{2}$; hence, $u_{*}=0$ in $\left[\tau_{1}, \tau_{2}\right]$, which is impossible because $x_{*}\left(\tau_{2}\right)=d\left(\tau_{2}\right)>e\left(\tau_{2}\right)$. The proofs of the other cases are analogous.

\section{Convergence}

Let $\left(x^{0}, u^{0}\right)$ be the unique solution of problem (2) with $\epsilon=0$; that is, $\left(x^{0}, u^{0}\right)$ is the solution of

$$
\begin{array}{ll}
\min & \|u\|, \\
\text { s.t. } & \ddot{x}(t)=u(t), \\
& x(a)=y_{a}, \quad \dot{x}(a)=s_{a}, \quad x(b)=y_{b}, \quad \dot{x}(b)=s_{b}, \\
& e(t) \leq x(t) \leq d(t), \quad \text { for all } t \in[a, b], u \in L^{2}[a, b] .
\end{array}
$$

Our first convergence result is given in the following theorem.

Theorem 3.1. Let $u_{\epsilon}$ be the optimal control of problem (2). Then,

$$
\lim _{\epsilon \rightarrow 0}\left\|u_{\epsilon}-u^{0}\right\|=0 \text {. }
$$

Proof. Let $\epsilon_{k}$ be a sequence of positive numbers convergent to zero and such that

$$
\begin{array}{r}
\epsilon_{k}<\min \left\{(1 / 8) \min _{t \in[a, b]}(d(t)-e(t)),(1 / 2)\left(\min _{i}\left(t_{i+1}-t_{i}\right) / 8\right)^{6}\right\}, \\
k=1,2, \ldots \mu,
\end{array}
$$


where $\mu=\min \left\{d\left(t_{i}\right)-x^{0}\left(t_{i}\right), x^{0}\left(t_{i}\right)-e\left(t_{i}\right)\right\}$, for all $i$ such that

$$
e\left(t_{i}\right)<x^{0}\left(t_{i}\right)<d\left(t_{i}\right) \text {. }
$$

For each $\epsilon_{k}$, we will find an admissible control $\tilde{u}_{\epsilon_{k}}$ for problem (2) with $\epsilon=$ $\epsilon_{k}$ such that

$$
\lim _{k \rightarrow \infty}\left\|u^{0}-\tilde{u}_{\epsilon_{k}}\right\|=0 .
$$

Suppose that $x^{0}\left(t_{i}\right)=d\left(t_{i}\right)$ for some $i$, where $x^{0}$ is the optimal output corresponding to $u^{0}$. Let

$$
p_{k}^{i}(t)= \begin{cases}-\left(t-t_{i}-\delta_{k}\right)^{3}\left(t-t_{i}+\delta_{k}\right)^{3}, & t \in\left[t_{i}-\delta_{k}, t_{i}+\delta_{k}\right], \\ 0, & \text { otherwise, }\end{cases}
$$

where $\delta_{k}^{6}=2 \epsilon_{k}$. If $x^{0}\left(t_{i}\right)=e\left(t_{i}\right)$ for some $i$, we take $p_{k}^{i}$ with negative sign. Let $p_{k}=\sum_{i} p_{k}^{i}$, where the summation is for all $i$ such that either $e$ or $d$ is active at $t_{i}$. Note that $p_{k}(t)$ is a $C^{2}$ function and $\left\|\ddot{p}_{k}\right\|_{L^{2}[a, b]} \rightarrow 0$ as $\epsilon_{k} \rightarrow 0$. Let $\tilde{x}_{k}=x^{0}+p_{k}$. Then,

$$
\lim _{k \rightarrow \infty}\left\|\ddot{\tilde{x}}_{k}-u^{0}\right\|=0
$$

and

$$
\tilde{x}_{k}\left(t_{i}\right)=d\left(t_{i}\right)-2 \epsilon_{k}<d\left(t_{i}\right)-\epsilon_{k}
$$

or

$$
\tilde{x}_{k}\left(t_{i}\right)=e\left(t_{i}\right)+2 \epsilon_{k}>e\left(t_{i}\right)+\epsilon_{k},
$$

depending on which of the constraints is active at $t_{i}$. Since the feasible set of problem (2) is contained in the feasible set of problem (6), we obtain

$$
\left\|u_{\epsilon_{k}}\right\| \leq\left\|\tilde{u}_{\epsilon_{k}}\right\|=\left\|u^{0}+\ddot{p}_{k}\right\| \rightarrow\left\|u^{0}\right\|, \quad \text { as } k \rightarrow \infty .
$$

From the above relations, we obtain

$$
\lim _{k \rightarrow \infty}\left\|u_{\epsilon_{k}}\right\|=\left\|u^{0}\right\|
$$

Moreover, the sequence $u_{\epsilon_{k}}, k=1,2, \ldots$, is bounded; therefore, $u_{\epsilon_{k}}$ has an $L^{2}$-weak cluster point, which we denote by $\bar{u}$. If $u_{l} \rightarrow \bar{u}$ weakly as $l \rightarrow \infty$, then by the weak lower semicontinuity of the $L^{2}$-norm, we obtain

$$
\left\|u^{0}\right\| \leq\|\bar{u}\| \leq \lim \inf _{l \rightarrow \infty}\left\|u_{l}\right\|=\left\|u^{0}\right\| .
$$

Hence, $u^{0}=\bar{u}$. From the uniqueness of $u^{0}$, there exists a unique $L^{2}$-weak cluster point of the sequence $u_{\epsilon_{k}}$ which is $u^{0}$. Since $u_{\epsilon_{k}} \rightarrow u^{0} L^{2}$-weakly and $\left\|u_{\epsilon_{k}}\right\| \rightarrow\left\|u^{0}\right\|$ as $k \rightarrow \infty$, we obtain

$$
\left\|u_{\epsilon_{k}}-u^{0}\right\| \rightarrow 0, \quad \text { as } k \rightarrow \infty .
$$


The above result yields that the solution $\left(x^{0}, u^{0}\right)$ of problem (6) has the following regularity properties.

Corollary 3.1. The optimal control $u^{0}$ of problem (6) is a piecewiselinear function in $[a, b]$. In every interval $\left(t_{i}, t_{i+1}\right)$, the optimal output $x^{0}$ has the following properties:

(P1) the constraints are not active;

(P2) there is one contact point that is either a single touching point or an end of a subarc whose other end is a fixed knot;

(P3) there are two contact points that either form a touching pair or are the ends of a subarc.

In contrast to problem (3), in this case it is possible that the fixed knots and the contact points form two subarcs in the same closed interval $\left[t_{i}, t_{i+1}\right]$, one for each of the constraints, or form a touching point on one constraint and a subarc on the other.

Our next result concerns the convergence of the optimal controls of a sequence of approximating problems (6) to the optimal control of problem (1).

Theorem 3.2. Let $e_{n}$ and $d_{n}$ be two sequences of piecewise linear and continuous functions such that $e_{n} \rightarrow g$ and $d_{n} \rightarrow f$ uniformly in the interval $[a, b]$. Let $u_{0}$ be the optimal control for problem (1), and let $u_{n}$ be the optimal controls for the approximating problems (6) with $e=e_{n}$ and $d=d_{n}$. Then,

$$
\lim _{n \rightarrow \infty}\left\|u_{n}-u_{0}\right\|=0 \text {. }
$$

Proof. Let $\tilde{u}$ be a feasible control for problem (1) such that the corresponding trajectory $\tilde{x}$ satisfies the boundary conditions (1c) and $g(t)<\tilde{x}(t)<f(t)$ for all $t \in[a, b]$. For any sequence $\delta_{n} \rightarrow 0$, if $\bar{u}_{n}=$ $u_{0}+\delta_{n}\left(\tilde{u}-u_{0}\right)$, then the corresponding output $\bar{x}_{n}$ satisfies (1c) and $g(t)<\bar{x}_{n}(t)<f(t)$ for all $t \in[a, b], \bar{x}_{n} \rightarrow x_{0}$ uniformly in $[a, b]$, where $x_{0}$ is the optimal output of problem (1). We will choose $\delta_{n}$ such that

$$
e_{n}(t) \leq \bar{x}_{n}(t) \leq d_{n}(t)
$$

for every sufficiently large $n$ and for all $t \in[a, b]$. Let $\alpha$ be the minimal distance from $\tilde{x}$ to the constraints, that is,

$$
0<\alpha=\min \left\{\min _{a \leq t \leq b}|\tilde{x}(t)-g(t)|, \min _{a \leq t \leq b}|\tilde{x}(t)-f(t)|\right\}
$$


and let

$$
T=\left\{t \in[a, b]:\left|x_{0}(t)-\tilde{x}(t)\right| \leq \alpha / 2\right\} .
$$

Let $\delta_{n}$ be a sequence of positive numbers convergent to zero and such that

$$
\delta_{n} \geq(2 / \alpha) \max \left\{\sup _{t \in[a, b]}\left|e_{n}(t)-g(t)\right| \sup _{t \in[a, b]}\left|d_{n}(t)-f(t)\right|\right\} .
$$

Let $t \in T$. We have

$$
\begin{aligned}
\bar{x}_{n}(t)-e_{n}(t) & =x_{0}(t)+\delta_{n}\left(\tilde{x}(t)-x_{0}(t)\right)-e_{n}(t) \\
& =x_{0}(t)-\tilde{x}(t)+\delta_{n}\left(\tilde{x}(t)-x_{0}(t)\right)+\tilde{x}(t)-g(t)+g(t)-e_{n}(t) \\
& \geq-\alpha / 2+\delta_{n}\left(\tilde{x}(t)-x_{0}(t)\right)+\alpha+g(t)-e_{n}(t) \geq 0,
\end{aligned}
$$

for $n$ sufficiently large. Analogously,

$$
\begin{aligned}
\bar{x}_{n}(t)-d_{n}(t) & =x_{0}(t)-\tilde{x}(t)+\delta_{n}\left(\tilde{x}(t)-x_{0}(t)\right)+\tilde{x}(t)-f(t)+f(t)-d_{n}(t) \\
& \leq \alpha / 2+\delta_{n}\left(\tilde{x}(t)-x_{0}(t)\right)-\alpha+f(t)-d_{n}(t) \leq 0,
\end{aligned}
$$

for $n$ sufficiently large. Let $t \notin T$. Consider first the case $x_{0}(t)-\tilde{x}(t)>\alpha / 2$. We have

$$
\begin{aligned}
\bar{x}_{n}(t)-e_{n}(t) & =x_{0}(t)-\tilde{x}(t)+\delta_{n}\left(\tilde{x}(t)-x_{0}(t)\right)+\tilde{x}(t)-g(t)+g(t)-e_{n}(t) \\
& \geq \alpha / 2+\delta_{n}\left(\tilde{x}(t)-x_{0}(t)\right)+\alpha+g(t)-e_{n}(t) \geq 0,
\end{aligned}
$$

for $n$ sufficiently large. Furthermore,

$$
\begin{aligned}
\bar{x}_{n}(t)-d_{n}(t) & =x_{0}(t)-f(t)+\delta_{n}\left(\tilde{x}(t)-x_{0}(t)\right)+f(t)-d_{n}(t) \\
& \leq \delta_{n}\left(\tilde{x}(t)-x_{0}(t)\right)+f(t)-d_{n}(t) \leq 0,
\end{aligned}
$$

because of the choice of $\delta_{n}$ in (8). Finally, let $x_{0}(t)-\tilde{x}(t)<-\alpha / 2$. Then,

$$
\begin{aligned}
\bar{x}_{n}(t)-e_{n}(t) & =x_{0}(t)-g(t)+\delta_{n}\left(\tilde{x}(t)-x_{0}(t)\right)+g(t)-e_{n}(t) \\
& \geq \delta_{n}\left(\tilde{x}(t)-x_{0}(t)\right)+g(t)-e_{n}(t) \geq 0,
\end{aligned}
$$

because of the choice of $\delta_{n}$ in (8), and

$$
\begin{aligned}
\bar{x}_{n}(t)-d_{n}(t) & =x_{0}(t)-\tilde{x}(t)+\delta_{n}\left(\tilde{x}(t)-x_{0}(t)\right)+\tilde{x}(t)-f(t)+f(t)-d_{n}(t) \\
& \leq-\alpha / 2+\delta_{n}\left(\tilde{x}(t)-x_{0}(t)\right)-\alpha+f(t)-d_{n}(t) \leq 0,
\end{aligned}
$$

for $n$ sufficiently large. Thus, (7) is proved. This yields that $\bar{u}_{n}$ is a feasible control for the approximating problem (6). From optimality, we have

$$
\left\|u_{n}\right\| \leq\left\|\vec{u}_{n}\right\| \rightarrow\left\|u_{0}\right\|, \quad \text { as } n \rightarrow \infty .
$$


The sequence $u_{n}$ is bounded in $L^{2}$; hence, one can extract a subsequence which converges $L^{2}$-weakly to some $\hat{u}$. The sequence of optimal outputs $x_{n}$ converges uniformly to the corresponding output $\hat{x}$, which clearly satisfies the constraints of (1). Passing to a subsequence and using the weak lower semicontinuity of the $L^{2}$-norm, we have

$$
\left\|u_{0}\right\| \leq\|\hat{u}\| \leq \lim \inf _{n_{i} \rightarrow \infty}\left\|u_{n_{i}}\right\| \leq \lim _{n_{i} \rightarrow \infty}\left\|\bar{u}_{n_{i}}\right\|=\left\|u_{0}\right\| .
$$

The uniqueness of the optimal control $u_{0}$ implies that $u_{n} \rightarrow u_{0} L^{2}$-weakly, hence in the norm of $L^{2}[a, b]$.

\section{Solving the Finite-Dimensional Problem}

First, we discuss the properties of the function $\Psi(s, y)$ defined by problem (3) for $s=\left[s_{1}, \ldots, s_{n-1}\right] \in \mathbb{R}^{n-2}$ and for $y=\left[y_{1}, \ldots, y_{n-1}\right] \in \mathbb{R}^{n-1}$, such that

$$
e\left(t_{i}\right)+\epsilon \leq y_{i} \leq d\left(t_{i}\right)-\epsilon, \quad i=1, \ldots, n-1 .
$$

Let us recall that a function $f$ is called coercive if $f(s) \rightarrow \infty$ as $|s| \rightarrow \infty$.

Theorem 4.1. The function $\Psi$ is convex, and coercive in $s$ uniformly in $y$; i.e., if $|s| \rightarrow \infty$, then $\Psi(s, y) \rightarrow \infty$ uniformly in $y \in \mathbb{R}^{n-2}$, $e\left(t_{i}\right)+\epsilon \leq y_{i} \leq d\left(t_{i}\right)-\epsilon, i=1, \ldots, n-1$. Moreover, $\Psi$ attains its unique minimum in its domain.

Proof. The convexity of $\Psi$ follows from the fact that $\Psi$ is the value function of a convex minimum problem. Let

$$
s=\lambda s^{1}+(1-\lambda) s^{2}, \quad y=\lambda y^{1}+(1-\lambda) y^{2}, \quad 0 \leq \lambda \leq 1 ;
$$

let $u^{1}$ be the optimal control for problem (3) corresponding to $\left(s^{1}, y^{1}\right)$; and let $u^{2}$ be the optimal control for problem (3) corresponding to $\left(s^{2}, y^{2}\right)$. Then, $\lambda u^{1}+(1-\lambda) u^{2}$ is an admissible control for problem (3) corresponding to $(s, y)$. Since the functional $I(u)$ defined in $(3)$ is convex, we obtain

$$
\begin{aligned}
\Psi\left(\lambda s^{1}+(1-\lambda) s^{2}, \lambda y^{1}+(1-\lambda) y^{2}\right) & \leq I\left(\lambda u^{1}+(1-\lambda) u^{2}\right) \\
& \leq \lambda I\left(u^{1}\right)+(1-\lambda) I\left(u^{2}\right) \\
& =\lambda \Psi\left(s^{1}, y^{1}\right)+(1-\lambda) \Psi\left(s^{2}, y^{2}\right),
\end{aligned}
$$

that is, $\Psi$ is convex. 
Let $u(s, y)$ denote the optimal control in problem (3). Using the Cauchy inequality, we have, for $i=0,1, \ldots, n-1$,

$$
\begin{aligned}
\left|s_{i+1}-s_{i}\right| & \leq \int_{t_{i}}^{t_{i+1}}|u(s, y ; t)| d t \\
& \leq\|u(s, y)\|_{L^{2}\left[t_{i,} t_{i+1}\right]}\left|t_{i+1}-t_{i}\right| \\
& \leq\|u(s, y)\||b-a| .
\end{aligned}
$$

Since $s_{0}=s_{a}, s_{n}=s_{b}$ are fixed, it follows that, if $|s| \rightarrow \infty$, then $\|u(s, y)\| \rightarrow \infty$ uniformly in $y$.

The minimum of problem (2), where the data $y_{i}$ satisfy $e\left(t_{i}\right)+\epsilon \leq y_{i} \leq d\left(t_{i}\right)-\epsilon, i=1, \ldots, n-1$, exists and is unique; hence, $\Psi$ attains its unique minimum in its domain. This proves the theorem.

The following corollary shows that problem (4) is well-suited for numerical computations.

Corollary 4.1. Problem (4) is Tikhonov well-posed; that is, every minimizing sequence converges to the unique solution.

Proof. Let $\left(s_{k}, y_{k}\right)$ be a minimizing sequence; that is,

$$
\Psi\left(s_{k}, y_{k}\right) \leq \Psi\left(s^{0}, y^{0}\right)+\delta_{k},
$$

where $\left(s^{0}, y^{0}\right)$ is the solution of problem (4) and $\delta_{k} \rightarrow 0$ as $k \rightarrow \infty$. The sequence $y_{k}$ is bounded and, by the coercivity of $\Psi$ with respect to $s$, it follows that the sequence $s_{k}$ is bounded as well. Without loss of generality, assume that $\left(y_{k}, s_{k}\right) \rightarrow(\bar{y}, \bar{s})$. Then, from the continuity of $\Psi$ and the uniqueness of the optimal solution $\left(s^{*}, y^{*}\right)$, we obtain that $(\bar{y}, \bar{s})=\left(y^{*}, s^{*}\right)$. This proves the Tikhonov well-posedness.

For a discussion of Tikhonov well-posedness and related topics, see Ref. 12, Chapter 1.

In our algorithm, we evaluate the function $\Psi$ in the following way:

$$
\Psi(s, y)=\sum_{i=0}^{n-1} \phi_{i}\left(s_{i}, s_{i+1}, y_{i}, y_{i+1}\right) .
$$


Here,

$$
\begin{aligned}
& \phi_{i}\left(s_{i}, s_{i+1}, y_{i}, y_{i+1}\right) \\
&=\min \|u\|_{\left.L^{2} t_{i}, t_{i+1}\right]}^{2}, \\
& \text { s.t. } \quad \ddot{x}(t)=u(t), \quad x\left(t_{i}\right)=y_{i}, \\
& \dot{x}\left(t_{i}\right)=s_{i}, \quad x\left(t_{i+1}\right)=y_{i+1}, \quad \dot{x}\left(t_{i+1}\right)=s_{i+1}, \\
& e(t) \leq x(t) \leq d(t), \quad \text { for all } t \in\left[t_{i}, t_{i+1}\right], u \in L^{2}\left[t_{i}, t_{i+1}\right] .
\end{aligned}
$$

Each of problems (10) can be solved explicitly as described below.

We say that an output of problem (10) for a given interval $\left[t_{i}, t_{i+1}\right]$ is admissible if it is a $C^{2}$ piecewise-cubic polynomial that has one of the four properties described in Theorem 2.1. The optimal output of problem (10) is an admissible output with the minimal $L^{2}$-norm of the second derivative. The construction of an admissible output reduces to a root-finding problem, where the unknowns are the coefficients of the cubic polynomials and the location of the contact points.

Further analysis of the admissible outputs in problem (10) yields that, if there is an admissible output with no contact points, then it is the optimal output. If there is an admissible output that has a subarc on a given constraint, then there is no admissible output that has a touching point on the same constraint. Conversely, if there is an admissible output that has a touching point on a given constraint, then there is no admissible output that has a subarc on the same constraint. If there exists an admissible output in problem (10) that has a subarc on one constraint, then there is no admissible output that has a subarc on the other constraint. Suppose there is no admissible output that has no contact points. In this case, if there exists an admissible output that has a touching point or a subarc, then the optimal output in problem (10) must have either a touching point or a subarc, and there is no need to consider the case of a touching pair. Proof of these statements can be obtained by a simple analysis based on properties of cubic polynomials.

Admissible outputs in problem (10) for each of the cases of a touching point, a subarc, or a touching pair are determined from the condition that the output is $C^{2}$ inside the interval, from the constraints (10b)-(10d), and by using the definition of a touching point, a subarc, or a touching pair, respectively. The location of a single touching point on a given constraint is determined from a cubic equation. Since a cubic equation has at most three roots, there exist at most three admissible outputs that have a touching point on a given constraint. The location of a subarc on a given constraint is determined by solving a linear equation. Hence, there exists at most one admissible output in problem (10) that has a subarc on a given constraint. 
The case of a touching pair reduces to two coupled polynomial equations for the locations of the contact points comprising the touching pair.

\section{Discontinuous and Piecewise Cubic Constraints}

In problem (2), we assume that the functions $e$ and $d$ are piecewise linear and continuous. This assumption can be relaxed so that the functions $e$ and $d$ need not be continuous at the fixed knots $t_{i}$ provided that there is a function $\zeta$ such that $\ddot{\zeta} \in L^{2}, \zeta(a)=y_{a}, \dot{\zeta}(a)=s_{a}, \zeta(b)=y_{b}, \dot{\zeta}(b)=s_{b}$, and the graph of $\zeta$ is in the interior of the closure of the set $\left\{(x, t) \in \mathbb{R}^{2}: e(t) \leq x \leq d(t)\right\}$. The existence of the function $\zeta$ guarantees, for $\epsilon$ sufficiently small, the existence and uniqueness of the solution to problem (2) and the nondegeneracy of the Lagrange multipliers. The characterizations of the solutions to problems (2) and (6) given in Corollaries 2.1 and 3.1 hold also for the case where the constraints $e$ and $d$ are discontinuous at $t_{i}$.

The above analysis can be also applied to a modification of problem (6) when, in each interval $\left[t_{i}, t_{i+1}\right]$, there is one constraint only which is a cubic polynomial. More precisely, let $I$ and $J$ be two disjoint subsets of the set $\{1,2, \ldots, n\}$, and let $\left\{e_{i}\right\}_{i \in I}$ and $\left\{d_{j}\right\}_{j \in J}$ be two families of cubic polynomials. Define

$$
e(t)= \begin{cases}e_{i}(t), & \text { if } i \in I \text { and } t \in\left(t_{i}, t_{i+1}\right] \\ -\infty, & \text { otherwise }\end{cases}
$$

and

$$
d(t)= \begin{cases}d_{j}(t), & \text { if } j \in J \text { and } t \in\left(t_{j}, t_{j+1}\right], \\ +\infty, & \text { otherwise. }\end{cases}
$$

Let $\left(t_{i}, y_{i}\right)$ be in the interior of the closure of the set $\left\{(x, t) \in \mathbb{R}^{2}: e(t) \leq x \leq d(t)\right\}$. In this case, from the necessary conditions for optimality (analogous to Lemma 2.1), it can be shown that the solution $x^{*}$ of problem (2) is a $C^{2}$ function which is a cubic polynomial in every interval $\left[\tau_{1}, \tau_{2}\right] \subset\left[t_{i}, t_{i+1}\right]$ where the constraints are not active. Let $x^{*}\left(\tau_{j}\right)=e\left(\tau_{j}\right)$, $j=1$, 2. Then, $\eta=f-e$ is a cubic polynomial with two zeros at $\tau_{1}$ and $\tau_{2}$ and $\eta>0$ on $\left(\tau_{i}, \tau_{i+1}\right)$. Since the function $\eta$ has minima at $\tau_{j}, \eta\left(\tau_{1}\right)=$ $\eta^{\prime}\left(\tau_{1}\right)=\eta\left(\tau_{2}\right)=\eta^{\prime}\left(\tau_{2}\right)=0$, which implies $\eta=0$. Hence, in each interval $\left[t_{i}, t_{i+1}\right]$, only a subarc or a touching point are possible. In this case, the optimal output for problem (2) is a $C^{2}$ piecewise-cubic polynomial, with perhaps a subarc (two contact points) or a touching point (one contact point) in every $\left[t_{i}, t_{i+1}\right]$. Note that subarcs may occur in two neighboring 
intervals. If in every interval $\left[t_{i}, t_{i+1}\right]$, both constraints $e$ and $d$ are cubic polynomials, then the optimal output may have up to four contact points in $\left[t_{i}, t_{i+1}\right]$.

\section{Numerical Examples}

For solving problem (4) numerically, we first choose an initial point $\left(s^{0}, y^{0}\right)$, e.g.,

$$
y_{i}^{0}=(1 / 2)\left(e\left(t_{i}\right)+d\left(t_{i}\right)\right), \quad s_{i}^{0}=f^{\prime}\left(t_{i}\right), \quad i=1, \ldots, n-1,
$$

where $f$ is the third-order $C^{2}$ Hermite spline satisfying

$$
\begin{array}{ll}
f\left(t_{0}\right)=y_{a}, & f^{\prime}\left(t_{0}\right)=s_{a}, \quad f\left(t_{b}\right)=y_{b}, \quad f^{\prime}\left(t_{b}\right)=s_{b}, \\
f\left(t_{i}\right)=y_{i}^{0}, & i=1, \ldots, n-1 .
\end{array}
$$

Then, we apply a code for finite-dimensional optimization. In our computations, we used the Optimization Toolbox and the Spline Toolbox of MATLAB Version 4.0. The numerical solution of problem (2) for the examples below takes several minutes on a Hewlett-Packard (HP) Workstation, 9000 Series, Model $715 / 50$ with a $50 \mathrm{MHz}$ PA-RISC 7100 Processor. Our experience shows that, if some of the fixed knots of the solution to problem (2) are close to the constraints, then the evaluation of $\Psi(s, y)$ may be numerically difficult. Although we have used a general-purpose minimization routine supplied by MATLAB, we feel that the performance of the algorithm can be increased by using optimization routines which take into account the specific structure of the problem. The development of such routines is a subject of continuing research.

Example 6.1. Our first example is to transfer the double integrator from the initial state

$$
x(0)=0, \quad \dot{x}(0)=-2
$$

to the final state

$$
x(25)=-1, \quad \dot{x}(25)=2,
$$

subject to

$$
e(t) \leq x(t) \leq d(t)
$$




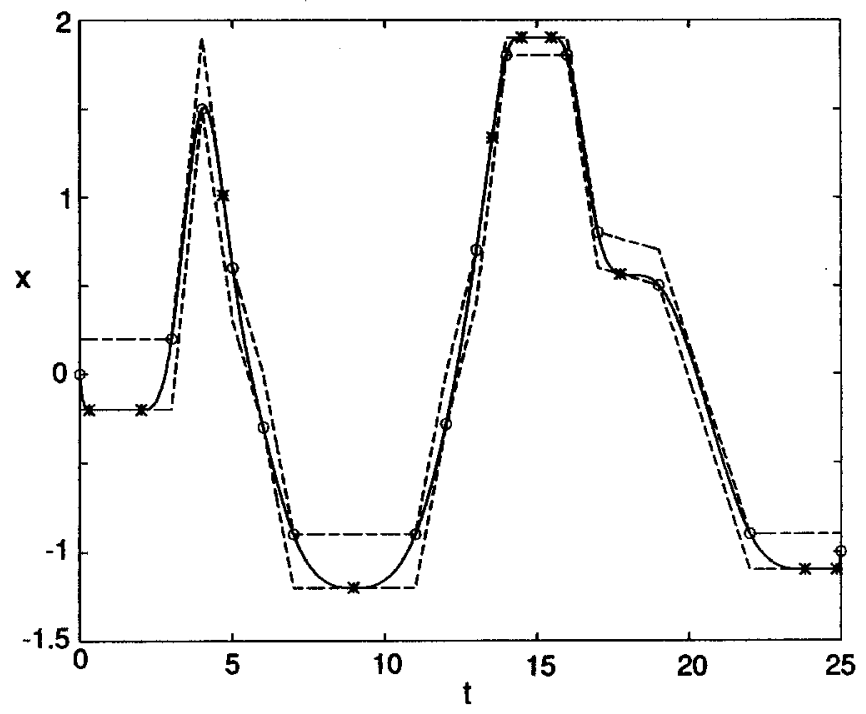

Fig. 2. Optimal output in Example 6.1: $O=$ fixed knot; $*=$ contact point.

where $e(t), d(t)$ are piecewise-linear and continuous functions shown by the dashed lines in Fig. 2. The optimal output of problem (2), obtained for $\epsilon=10^{-6}$, is shown by the solid line in Fig. 2. All but two fixed knots lie on an $\epsilon$-distance from the constraints and the output has three subarcs and

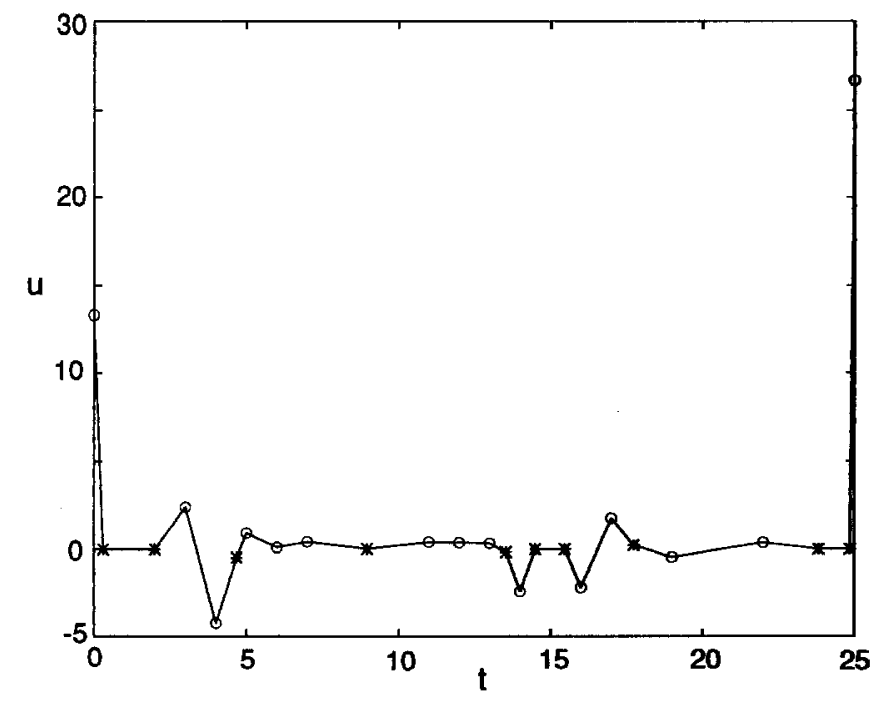

Fig. 3. Optimal control in Example 6.1: $O=$ fixed knot; $*=$ contact point. 


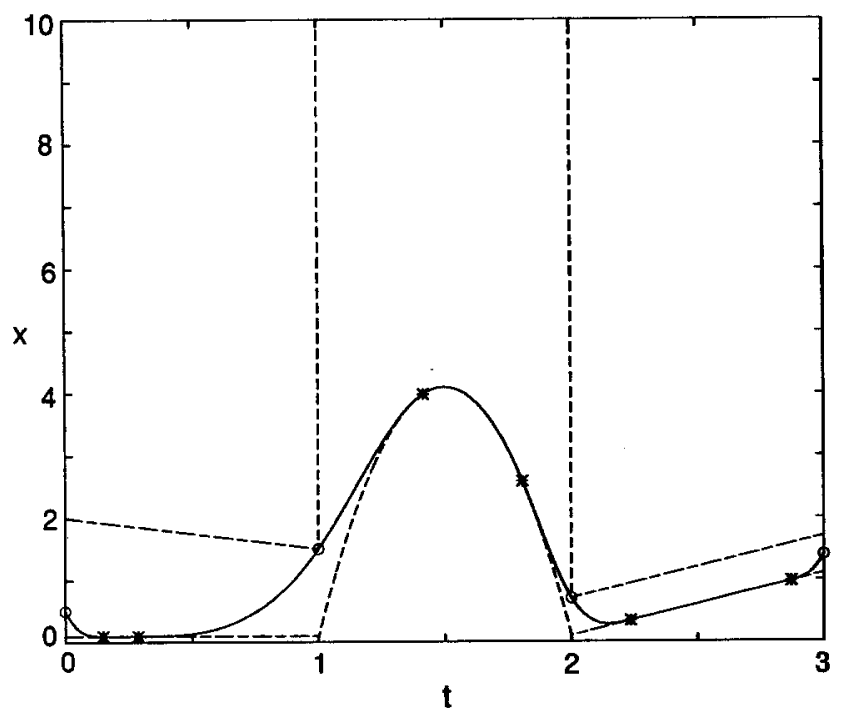

Fig. 4. Optimal output in Example 6.2: $\mathrm{O}=$ fixed knots; $*=$ contact point.

four touching points. The corresponding optimal control is shown in Fig. 3.

Example 6.2. In the second example, we have

$$
x(0)=0.5, \quad \dot{x}(0)=-8, \quad x(3)=1.4, \quad \dot{x}(3)=8,
$$

and the constraints are shown by the dashed lines in Fig. 4. The constraints are not continuous at the fixed knots; in the second interval, one of the constraints is a cubic polynomial, while the other constraint is absent. This example illustrates our approach for the more general problem described in Section 5. The optimal output of problem (2), obtained for $\epsilon=10^{-6}$, is shown by the solid line in Fig. 4. Figure 5 shows the optimal control.

\section{Appendix: Regularity of the Optimal Control of a State Constrained Linear Regulator Problem}

Consider the following problem with one-sided state constraint:

$$
\min (1 / 2) \int_{a}^{b}\left(x(t)^{T} Q x(t)+u(t)^{T} u(t)\right) d t
$$




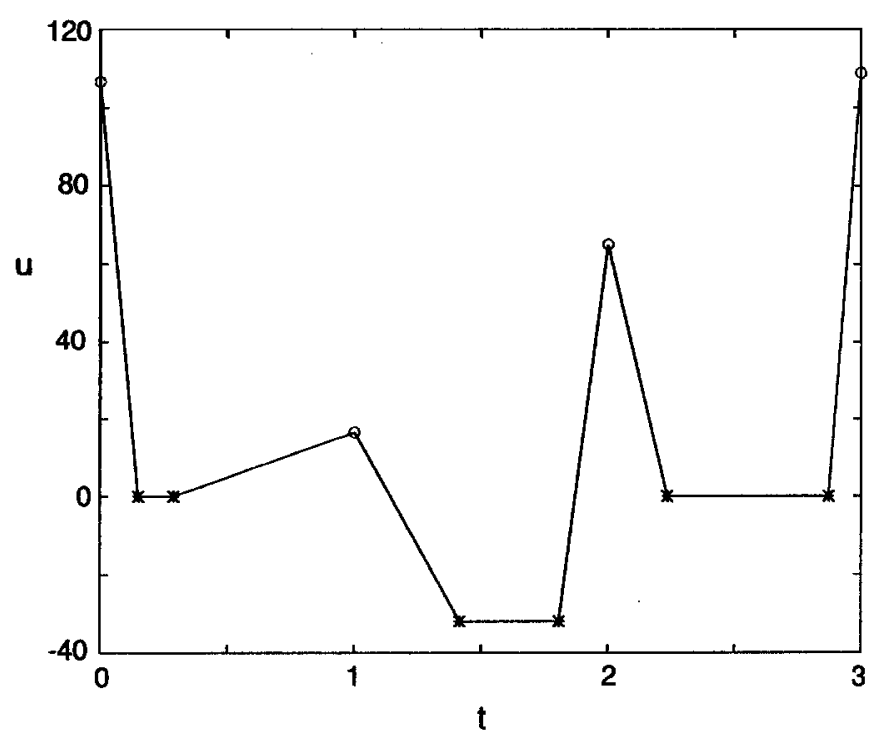

Fig. 5. Optimal control in Example 6.2: $\bigcirc=$ fixed knot; $*=$ contact point.

on the set of those $x \in W^{1,2}[a, b]$ and $u \in L^{2}[a, b]$ such that

$$
\begin{aligned}
& \dot{x}(t)=A x(t)+B u(t), \quad x(0)=x^{0}, \\
& l^{T} x(t)+c \leq 0, \quad \text { for all } t \in[a, b],
\end{aligned}
$$

where $x(t) \in \mathbb{R}^{n}, u(t) \in \mathbb{R}^{m}$, the matrices $Q, A, B$ are constant, $Q$ is symmetric and positive semidefinite, $l \in \mathbb{R}^{n}, c \in \mathbb{R}^{1}$, the initial state $x^{0}$ is fixed, and the superscript ${ }^{T}$ denotes transposition. From the analysis in Ref. 11, Chapter 2, it follows that, if $l^{T} B=0$ and $l^{T} A B \neq 0$, then the optimal control of problem (11) is a piecewise analytic function in time. It is also mentioned in Ref. 11 that the optimal control is analytic if $l^{T} B \neq 0$. We present here a simple proof of the latter assertion using Hager's regularity result (Ref. 10). We also discuss briefly the regularity properties of the optimal control when this condition is not satisfied.

Let us recall that, if for some trajectory $x$ the state constraint is active at $t$ (i.e., is satisfied as equality), and for every $\alpha>0$ it is nonactive somewhere in $[t-\alpha, t+\alpha]$, then $t$ is a contact point of $x$.

Theorem 7.1. Suppose that $l^{T} x^{0}+b<0$ and the vector

$$
l^{T} B \neq 0 \text {. }
$$

Then, the optimal control is piecewise analytic in $[a, b]$. 
We start with the following simple lemma:

Lemma 7.1. There exists a control $\tilde{u}$ such that, if $\tilde{x}$ is the corresponding solution of (12a), then $l^{T} \tilde{x}(t)+b<0$ for all $t \in[a, b]$.

Proof. From (13),

$$
\beta=l^{T} B B^{T} l>0 .
$$

Let $\tilde{x}$ be the solution of

$$
\dot{x}(t)=\left(A-B B^{T} l l^{T} A / \beta\right) x(t), \quad x(0)=x^{0},
$$

and let

$$
\tilde{u}(t)=-B^{T} l l^{T} A \tilde{x}(t) / \beta .
$$

Then,

$$
l^{T} \dot{\tilde{x}}=\left(l^{T} A-l^{T} B B^{T} l l^{T} A / \beta\right) \tilde{x}(t)=0,
$$

that is,

$$
l^{T} \tilde{x}(t)+c=l^{T} x^{0}+c<0, \quad \text { for all } t \in[a, b] .
$$

Since the set of feasible controls is nonempty, there exists a solution $(\hat{x}, \hat{u})$ to (11) and, by the strict convexity of the functional, the solution is unique. Furthermore, Lemma 7.1 implies that the first-order optimality conditions are in normal form (see Refs. 1-2); namely, there exist a function of bounded variation $\psi$ and a nonnegative regular measure $\mu$ supported on the set $\left\{t \in[a, b]: l^{T} \hat{x}(t)+c=0\right\}$ such that, for a.e. $t \in[a, b]$,

$$
\begin{aligned}
& \hat{u}(t)=B^{T} \psi(t), \\
& \psi(t)=\int_{t}^{b}\left[A^{T} \psi(\tau)-Q \hat{x}(\tau)\right] d \tau-\int_{t}^{b} l d \mu .
\end{aligned}
$$

Proof of Theorem 7.1. Condition (13) coincides with Hager's condition; hence, the nondecreasing function

$$
v(t)=-\int_{t}^{b} d \mu
$$

is Lipschitz continuous in the interval $[a, b)$. Then, from (14) and (15), we obtain that the optimal solution $(\hat{x}, \hat{u})$ satisfies the following relations, for 
a.e. $t \in[a, b]$ :

$$
\begin{aligned}
u(t) & =B^{T} \psi(t), \\
\dot{x}(t) & =A x(t)+B B^{T} \psi(t), \\
\dot{\psi}(t) & =-A^{T} \psi(t)+Q x(t)+l \dot{v}(t), \\
0 & =\left(l^{T} x(t)+c\right) \dot{v}(t) .
\end{aligned}
$$

Let

$$
y(t)=l^{T} \hat{x}(t)+c .
$$

Suppose that there exists an infinite sequence $\tau_{1}, \tau_{2}, \ldots, \tau_{k}, \ldots$ of contact points of $y$ in $[a, b]$. Without loss of generality, let $\tau_{k}$ converge to $t_{0} \in[a, b)$, and let $\tau_{1} \geq \tau_{2} \geq \ldots \geq \tau_{k} \ldots \geq t_{0}$. Let $y(t)<0$ on $\left(\tau_{2 k+1}, \tau_{2 k}\right)$ and $y(t)=0$ on $\left[\tau_{2 k+2}, \tau_{2 k+1}\right]$ for $k=1,2, \ldots$. Suppose that $\tau_{2 k+2}<\tau_{2 k+1}$ for infinitely many $k$ from a set of natural numbers $I$. Denote

$$
\begin{aligned}
& v=\left[\begin{array}{c}
x \\
\psi
\end{array}\right], \quad N=\left[\begin{array}{cc}
A & B B^{T} \\
Q & -A^{T}
\end{array}\right], \quad f=\left[\begin{array}{l}
0 \\
l
\end{array}\right], \\
& r(t)=\left[l^{T}, 0\right] \exp (N t) f, \quad z(t)=\left[l^{T}, 0\right] \exp \left(N\left(t-t_{0}\right)\right) v\left(t_{0}\right)+c .
\end{aligned}
$$

From (16b) and (16c), we obtain that, for every $t \in[a, b]$,

$$
y(t)=z(t)+\int_{t_{0}}^{t} r(t-\tau) \dot{v}(\tau) d \tau .
$$

Note that

$$
r(0)=0 \quad \text { and } \quad \dot{r}(t 0)=\beta=l^{T} B B^{T} l>0 .
$$

Since $\psi$ is absolutely continuous, one can differentiate (17) obtaining

$$
\dot{y}(t)=\dot{z}(t)+\int_{t_{0}}^{t} \dot{r}(t-\tau) \dot{v}(\tau) d \tau .
$$

From the continuity of $y$ and $\dot{y}$, it follows that

$$
y\left(t_{0}\right)=\dot{y}\left(t_{0}\right)=z\left(t_{0}\right)=\dot{z}\left(t_{0}\right)=0 .
$$

If $z$ is identically zero, then the above equation yields that, for $k \in I$ sufficiently large and for each $t \in\left(\tau_{2 k+2}, \tau_{2 k+1}\right)$,

$$
0=\int_{t_{0}}^{t} \dot{r}(t-\tau) \dot{v}(\tau) d \tau \geq(1 / 2 \beta)\left(v(t)-v\left(t_{0}\right)\right) \geq 0 .
$$


This implies that $v$ is constant on $\left[t_{0}, t_{0}+\alpha\right]$ for some $\alpha>0$, which in turn yields that $y=z$ in $\left[t_{0}, t_{0}+\alpha\right]$, a contradiction. Therefore, $z$ is not identically zero, which is equivalent to saying that there exists a natural number $j \geq 2$ such that the $j$ th derivative $z^{(j)}\left(t_{0}\right) \neq 0$ and $z^{i}\left(t_{0}\right)=0$ for $0 \leq i<j$.

Let $t \in\left(\tau_{2 k+2}, \tau_{2 k+1}\right)$. Then from (18) and using the Taylor expansion for $z$, for all sufficiently large $k \in I$, we have

$$
\begin{aligned}
0 & =\dot{z}(t)+\int_{t_{0}}^{t} \dot{r}(t-\tau) \dot{v}(\tau) d \tau \\
& \geq z^{(j)}\left(t_{0}\right)\left(t-t_{0}\right)^{j-1} /(j-1) !+o\left(\left(t-t_{0}\right)^{j-1}\right)+\beta \Delta v(t) / 2,
\end{aligned}
$$

where

$$
\Delta v(t)=v(t)-v\left(t_{0}\right) \geq 0 .
$$

Since $\Delta v$ is constant in $\left[\tau_{2 k+1}, \tau_{2 k}\right]$, then the above inequality implies that there exists $\gamma>0$ such that, for some sufficiently small $\alpha>0$ and for all $t \in\left[t_{0}, t_{0}+\alpha\right]$,

$$
0 \leq \Delta v(t) \leq \gamma\left(t-t_{0}\right)^{j-1} .
$$

Now, let $t \in\left(\tau_{2 k+1}, \tau_{2 k}\right)$. Since $\dot{v}=0$ on $\left(\tau_{2 k+1}, \tau_{2 k}\right)$, from (18) we get

$$
\dot{y}(t)=\dot{z}(t)+\int_{t_{0}}^{\tau_{2 k}} \dot{r}(t-\tau) \dot{v}(\tau) d \tau .
$$

Differentiating this equality and using the Taylor expansion for $z$, we obtain that, for some constant $\kappa>0$,

$$
\begin{aligned}
\ddot{y}(t) & =z^{j}\left(t_{0}\right)\left(t-t_{0}\right)^{j-2} /(j-2) !+\int_{t_{0}}^{\tau_{2 k}} \ddot{r}(t-\tau) \dot{v}(\tau) d \tau+o\left(\left(t-t_{0}\right)^{j-2}\right) \\
& \leq z^{(j)}\left(t_{0}\right)\left(t-t_{0}\right)^{j-2} /(j-2) !+\kappa \Delta v\left(\tau_{2 k}\right)+o\left(\left(t-t_{0}\right)^{j-2}\right) .
\end{aligned}
$$

Using (19), we conclude that for $t \in\left(\tau_{2 k}, \tau_{2 k+1}\right), k \in I$,

$$
\ddot{y}(t)=z^{j-2}\left(t_{0}\right)\left(t-t_{0}\right)^{j-2} /(j-2) !+o\left(\left(t-t_{0}\right)^{j-2}\right) .
$$

This means that, for all sufficiently large $k$, the function $\ddot{y}(t)$ has constant sign in the interval $\left(\tau_{2 k+1}, \tau_{2 k}\right)$, which contradicts $\dot{y}\left(\tau_{2 k+1}\right)=\dot{y}\left(\tau_{2 k}\right)=0$. Hence, $I$ is a finite set. Since $v$ is continuous, it is constant in $\left[t_{0}, t_{0}+\alpha\right]$ for some small $\alpha>0$. But then $y$ is analytic in $\left[t_{0}, t_{0}+\alpha\right]$, because $z$ is analytic there, and thus $y$ has a finite number of zeros. This means that the number of contact points is finite in the right neighborhood of $t_{0}$. The proof is complete. 
In the proof, we use a local argument; hence, the same conclusion can be obtained for several state constraints if one assumes that the condition (13) holds for every constraint, and at each instant of time no more than one of the constraints is active along the optimal trajectory. If there are additional control constraints, to apply the above proof one may assume that the state and control constraints do not have the same contact points.

Suppose that condition (13) is violated, that is, $l^{T} B=0$. Then from (14) and (15), we obtain

$$
\hat{u}(t)=\int_{t}^{b}\left(B^{T} A^{T} \psi(\tau)-B^{T} Q \hat{x}(\tau)\right) d \tau,
$$

that is, the optimal control $\hat{u}$ is differentiable almost everywhere in $[a, b]$ and its derivative is of bounded variation. If $l^{T} A B \neq 0$, we know from Ref. 11 that $\hat{u}$ is piecewise analytic. Now, suppose that $l^{T} A B=0$. Differentiating (20) and using (15), we obtain

$$
\dot{\hat{u}}=B^{T} Q \hat{x}(t)-B^{T} A^{T}\left[\int_{t}^{b}\left(A^{T} \psi(\tau)-Q \hat{x}(\tau) d \tau\right] .\right.
$$

This means that $\hat{u}$ is twice differentiable and its second derivative is of bounded variation. Proceeding in the same manner, we come to the following result.

Theorem 7.2. Suppose that, for some $0<k<n-1$,

$$
l^{T} A^{i} B=0, \quad \text { for } i=0,1, \ldots, k-1 .
$$

The optimal control $\hat{u}$ of problem (11) is $k$-differentiable in $[a, b]$ and its $k$ th derivative is of bounded variation.

Note that, if

$$
l^{T} A^{i} B=0, \quad \text { for } i=0,1, \ldots, n-1,
$$

then

$$
l^{T} x(t)=l^{T} \exp (A(t-a)) x^{0}, \quad \text { for all } t \in[a, b] ;
$$

that is, the violation of the state constraint cannot be controlled by $u$.

Considering a fourth-order integrator with a sign constraint for the first derivative (in this case, $l^{T} B=0, l^{T} A B=0$, but $l^{T} A^{2} B \neq 0$ ), Robbins has shown (Ref. 13) that the optimal trajectory may reach and leave the constraints infinitely many times; i.e., the optimal control may not be piecewise analytic. A similar example is analyzed in Ref. 11. As stated in Theorem 7.2, in this 
case the optimal control is twice differentiable; that is, by losing its piecewise analyticity, the optimal control becomes more smooth.

\section{References}

1. Hager, W. W., and Mitter, S. K., Langrange Duality Theory for Convex Control Problems, SIAM Journal of Control and Optimization, Vol. 14, pp. 843856, 1976.

2. Ioffe, A. D., and Tiknomirov, V. M., Theory of Extremal Problems, Nauka, Moscow, Russia, 1974.

3. Dontchev, A. L., Best Interpolation in a Strip, Journal of Approximation Theory, Vol. 73, pp. 334-342, 1993.

4. Budak, B. M., and Vasilev, F. P., Some Computational Aspects of Optimal Control Problems, Moscow University Press, Moscow, Russia, 1975 (in Russian).

5. Ermolev, Y. M., Gulenko, V. P., and Tsarenko, T. I., Finite-Element Methods in Optimal Control, Naukova Dumka, Kiev, Ukraine, 1978 (in Russian).

6. Fedorenko, R. P., Approximate Solution of Optimal Control Problems, Nauka, Moscow, Russia, 1971 (in Russian).

7. Gilbert, E. M., and Johnson, D. W., Distance Functions and Their Application to Robot Path Planning in the Presence of Obstacles, IEEE Journal of Robotics and Automation, Vol. 1, pp. 21-30, 1985.

8. Polak, E., Computational Methods in Optimization: A Unified Approach, Academic Press, New York, New York, 1971.

9. Dontchev, A. L., Perturbations, Approximations, and Sensitivity Analysis of Optimal Control Systems, Lecture Notes in Control and Information Science, Springer, Berlin, Germany, Vol. 52, 1983.

10. Hager, W. W., Lipschitz Continuity for Constrained Processes, SIAM Journal on Control and Optimization, Vol. 13, pp. 449-471, 1976.

11. Dikusar, V. V., and Milyutin, A. A., Qualitative and Numerical Methods in the Maximum Principle, Nauka, Moscow, Russia, 1989 (in Russian).

12. Dontchev, A. L., and ZolezzI, T., Well-Posed Optimization Problems, Lecture Notes in Mathematics, Springer, Berlin, Germany, Vol. 1543, 1993.

13. RobBins, H., Junction Phenomena for Optimal Control with State-Variable Inequality Constraints of Third Order, Journal of Optimization Theory and Applications, Vol. 31, pp. 85-99, 1980. 